\title{
Efficacy of Dietary Behavior Modification for Preserving Cardiovascular Health and Longevity
}

\author{
Moira McAllister Pryde and William Bernard Kannel \\ Framingham Heart Study, Boston University School of Medicine, 73 Mount Wayte Avenue, Framingham, MA 01702, USA \\ Correspondence should be addressed to Moira McAllister Pryde, mmpryde@bu.edu
}

Received 2 September 2010; Accepted 30 November 2010

Academic Editor: Demosthenes Panagiotakos

Copyright (C) 2011 M. M. Pryde and W. B. Kannel. This is an open access article distributed under the Creative Commons Attribution License, which permits unrestricted use, distribution, and reproduction in any medium, provided the original work is properly cited.

Cardiovascular disease (CVD) and its predisposing risk factors are major lifestyle and behavioral determinants of longevity. Dietary lifestyle choices such as a heart healthy diet, regular exercise, a lean weight, moderate alcohol consumption, and smoking cessation have been shown to substantially reduce CVD and increase longevity. Recent research has shown that men and women who adhere to this lifestyle can substantially reduce their risk of coronary heart disease (CHD). The preventive benefits of maintaining a healthy lifestyle exceed those reported for using medication and procedures. Among the modifiable preventive measures, diet is of paramount importance, and recent data suggest some misconceptions and uncertainties that require reconsideration. These include commonly accepted recommendations about polyunsaturated fat intake, processed meat consumption, fish choices and preparation, transfatty acids, low carbohydrate diets, egg consumption, coffee, added sugar, soft drink beverages, glycemic load, chocolate, orange juice, nut consumption, vitamin D supplements, food portion size, and alcohol.

\section{Introduction}

Healthy choices and behaviors influence CVD incidence and mortality. However, changing our dietary lifestyle in order to avoid cardiovascular disease is not easy. To succeed in reducing CVD, dietary alterations have to be maintained long term, which is a much more difficult task than taking pills. As a result, both patients and physicians gravitate toward medication for lowering CVD risk and prolonging life with only cursory attempts at changing lifestyle behaviors such as eating habits. There is the unfortunate perception that even with intensive counseling, risk factor modification by dietary means is trivial. Recent research indicates that this is not the case and that the necessary behavior modification regarding the diet can be achieved and confers a substantial benefit when implemented in conjunction with other behavior modification [1].

\section{Methods}

We performed a systematic review focused mainly on large trials with about 1900 subjects and meta-analyses of evidence for relationships of dietary factors with incident CHD, CVD, stroke, and diabetes mellitus. We searched for any cohort study, case-control study, or randomized trial that assessed these exposures and outcomes in generally healthy adults or subjects with overt CVD. We completed a Google search using keywords such as healthy lifestyle, healthy behaviors, CVD, CHD, stroke, and diabetes. Thus, this paper is based mainly on a 2000-2010 Internet search for well-designed relevant trials and data that confirm and quantify established dietary recommendations and novel nutritional data, shown or alleged to be alterable by changes in behavior and to be beneficial for CVD risk factor control.

\section{Results}

Despite decades of efforts to control them, half of USA adults still have unacceptable CVD risk factors. The latest NHANES survey (1999-2006) shows that $45 \%$ had one of the three major risk factors: $30.5 \%$ had hypertension, $26 \%$ high blood cholesterol, and $9.9 \%$ diabetes. About 13\% had two of the conditions and $3 \%$ had all three. The risk factor rates were higher in blacks and 15\% were unaware of having these 
conditions. The fraction of persons with one or more of these conditions and unaware of it was consistent across all ethnic groups. With $60 \%$ of adults and $30 \%$ of younger Americans obese, the CVD risk burden threatens to worsen. We appear to be headed for a generation with poorer health than the preceding one, which is unique [2].

Lifestyle, in general, and the diet we choose to eat, in particular, affects each of the major risk factors. A number of nutritional behaviors are recommended for avoiding cardiovascular risk factors and thereby prolonging our lifespan. In addition to recommendations for medicating hypertension, HDL-C, LDL-C, and diabetes, strong behavioral measures are now available for control of these major risk factors. In the Nurses' Health Study 82\% of CHD incidence occurred in those who failed to adhere to a sustained regimen that incorporates healthy dietary choices along with moderate exercise, maintaining a normal body weight, smoking abstinence, and moderate alcohol intake. Also, in the Health Professionals Follow-Up Study adherence to this healthy lifestyle reduced the lifetime risk of heart failure $[3,4]$.

3.1. Established Nutritional Factors. Evidence has now accumulated that quantifies and substantiates accepted recommendations for consumption of cholesterol, saturated, polyunsaturated, and transfats in the diet. In addition, new data suggest that some foods we were told to avoid may be acceptable if consumed in moderation.

3.1.1. Replacing Saturated Fat with Polyunsaturated Fat. People have been advised to avoid eating saturated fats to preserve CVD health for 60 years or more. However, there has been little evidence from research to substantiate this recommendation. The food industry and the general public have reduced saturated fat over the years, but have often replaced them with harmful trans fats and refined carbohydrates. A recent study by the Harvard School of Public Health has produced the first conclusive evidence that reducing saturated fat with polyunsaturated fat substitution reduces CHD risk by $19 \%$. The meta-analyses of 8 randomized trials revealed that for every $5 \%$ increase in polyunsaturated fat consumed, the risk of CVD decreased by $10 \%$. The Institute of Medicine recommends that $5 \%-10 \%$ of energy intake should come from polyunsaturated fats. Other trials have advised an intake of 15\% [5].

3.1.2. Processed versus Unprocessed Red Meats. Probing of the influence of saturated fat and cholesterol in the diet has now been examined from the real-world perspective of the marketplace. For example, a unique meta-analysis of globally obtained data reported an examination of the relationship between eating processed as opposed to unprocessed red meats and the risk of CHD and diabetes. The analyses indicated a direct relationship between consumption of processed meats and the risk of $\mathrm{CHD}$ and diabetes. After adjustment for CHD risk factors, the risk of CHD increased by $42 \%$ and the risk of diabetes increased by $19 \%$ for every $50 \mathrm{gm}$ of processed meat consumed daily. Processed meats, for example, bacon, sausages, or luncheon meats are preserved by adding salt, smoking, curing, or by adding preservatives. A $50 \mathrm{gm}$ serving would be equivalent to a hot dog or 1-2 slices of processed meat. The amount of cholesterol and saturated fat contained in both processed and unprocessed meat is comparable. However, the amount of sodium in processed meats is four times greater than that of unprocessed and it has 50\% more nitrate preservatives. The results of this study suggest that it is the sodium and preservatives in processed red meats, and not the fats that increase its risk of CVD and diabetes. Dietary salt does increase blood pressure, and studies of animals suggest that nitrate preservatives contribute to atherosclerosis and decrease glucose intolerance; conditions that are known to raise CHD and diabetes risk [6]. However, it is not established that processed meat confers a higher CHD risk than unprocessed meat.

3.1.3. Hazards of Red, White, and Processed Meats. A study of the relationship between white, red, and processed meat with mortality in half a million middle-aged participants of the NIH-AARP Diet \& Health Study was recently reported by Sinha et al. Consumption of processed and red meats was found to increase total deaths, cancer, and CVD deaths, whereas white meat protected against total mortality and cancer mortality. Red meat appeared to increase all the specified mortalities more than the processed meats. Red meat increased overall mortality $31 \%$ in men and $35 \%$ in women, whilst processed meat increased it $15 \%$ and $25 \%$ in men and women, respectively. For cancer mortality there was a $27 \%$ increase for red meat for men and $50 \%$ increase for women, whereas processed meat conferred a lesser $12 \%$ increase in men and $11 \%$ in women. For CVD, red meat imposed a $27 \%$ increase in men and $50 \%$ in women, while for processed meat the increases were $9 \%$ in men and 38\% in women [7].

Unlike meat products that are high in fat, fish is low in saturated fats and is a good source of protein and omega-3 fatty acids. Omega-3 fatty acid has been linked to a reduction in the development of atherosclerotic plaque, triglyceride levels, blood pressure, and the risk of arrhythmias. Consumption of fatty fish high in omega- 3 fatty acids such as salmon, sardines, and tuna at least twice per week is recommended for preserving cardiovascular health. Furthermore, Omega-3 fatty acid intake from food rather than from supplements is advisable. However, for persons with established coronary disease it may be advisable to also prescribe a supplement of omega-3 [8].

Consideration of the type of fish to be consumed as well as the means of preparation is also necessary. Due to the high levels of contaminants and mercury contained in some larger fish, such as swordfish and king mackerel, the USA Food and Drug Administration recommend that children, expectant women, and nursing mothers avoid consumption of these fish and opt for fish that are lower in mercury such as, salmon, catfish, and canned light tuna. For the middleaged and the elderly, eating an assortment of fish to help diminish the detrimental effects of potential impurities is recommended [8]. The cardioprotective benefits of eating fish are dependent not only on the quantity and the type 
of fish consumed, but also the way that it is prepared. Meng et al. found that men who ate the most fish had a $23 \%$ decrease in coronary heart disease and those who ate adequate amounts of baked or boiled fish had a lower coronary disease death rate than those who ate little fish. On the other hand, those who ate deep fried, salted, and dried fish daily had an increase in CHD mortality of $12 \%-15 \%$ [9].

$\mathrm{Hu}$ et al. provide evidence supporting recommendations to eat fish for cardioprotection. They found that 84,000 women nurses enrolled in the Nurses' Health study who consumed higher amounts of fish and omega-3 fatty acid supplements have a reduced risk of $C H D$. Within a 16 year observational period, 1,513 cases of incident CHD occurred, 484 CHD mortalities and 1,029 nonfatal myocardial infarctions. Women who consumed a higher amount of fish had a lower risk of coronary disease compared to those who ate fish less than once per month. A comparable result was found for those who consumed higher amounts of omega -3 fatty acids. The protective relationship appeared to be more powerful for coronary mortality than for nonfatal myocardial infarction [10].

Contrary to the aforementioned research studies and recommendations, Nair and Connolly did not find convincing evidence to support the recommendation for routine consumption of omega- 3 fatty acids. They reported that Jenkins and colleagues failed to demonstrate a convincing beneficial effect for prevention of arrhythmia and concluded that cited meta-analyses had provided weak evidence that omega-3 fatty acid intake can prevent either ventricular arrhythmia or cardiovascular mortality [11].

3.1.4. Egg Consumption. As early as 1982 Dawber et al. of the Framingham study reported on the nutrient intake of a subsample of Framingham study participants that allowed an estimate of egg consumption on 912 subjects. It was concluded that within the range of egg intake of this general population sample egg consumption is unrelated to serum cholesterol or to CHD incidence [12].

In $1999 \mathrm{Hu}$ et al. reported findings from the Health Professionals Study and Nurses' Health Study that prospectively examined consumption of eggs as a risk for initial CVD events in subjects free of high cholesterol, diabetes, or cancer. There were 1805 initial CHD and 821 first strokes in 8 years. Adjusting for age, smoking, and other risk factors, they found no significant link between 1-6 eggs weekly and coronary heart disease or stroke risk in either sex. However, egg use was associated with a statistically significant 1.52 -fold increased coronary disease risk in diabetic men and women on comparison of consumption of more than 1 egg daily versus less than 1 egg per week. They concluded that eating one or fewer eggs per day has little impact on risk of coronary disease or stroke in healthy persons but that egg consumption in diabetics warrants further study [13].

A later study of egg consumption in the Physicians' Health Study by Djoussé et al. in 2008 examined the relationship between egg intake and the risk of CVD events and mortality prospectively in 21,327 participants. Over the 20 year period, there were 8061 new events; 19\% of which were new myocardial infarctions, $17 \%$ initial strokes, and $64 \%$ deaths. After adjustment for other risk factors multivariable analysis showed that consumption of eggs was unrelated to incident myocardial infarction or stroke, however with respect to mortality there is a modest relationship particularly in diabetics where the risk was increased twofold $(P$ for interaction $=0.09)$. It does not appear that physicians need to restrict moderate egg consumption in patients, unless they are diabetic [14].

3.2. Novel Dietary Factors. Recent research of modifiable elements of behavior has reported findings that are contradictory to some common perceptions about nutrition. Many lifestyle elements that were once believed to be detrimental to health do not appear to be damaging and in some cases are beneficial. Among the novel factors reported in recent studies are consumption of coffee, dark chocolate, nuts, orange juice and vitamin $\mathrm{D}$.

3.2.1. Coffee Consumption. There has been a long standing controversy about the effect of coffee on CVD risk with early studies finding it harmful and recent ones judging it helpful or harmless. Until recently, studies have reported that coffee lovers risk having excess cardiac rhythm disturbances as well as myocardial infarctions and coronary heart disease in general. In a review of coffee consumption and cardiovascular disease in 1989 Wilson et al. called attention to a prospective study of 45,589 subjects that reported only a trivial increase in CVD risk for persons who consume more than 4 cups of coffee per day, whereas there was a strong positive correlation between decaffeinated coffee and the risk of CHD [15].

More recent large studies have reported that coffee actually does not increase CVD risk and may even decrease the rate of CVD events. These include the Nurses' Health Study that reported no association between coffee consumption and CVD occurrence in a study of 85,747 women who were followed for 10 years, a Swedish study found no association between coffee and post-MI death and an inverse relationship between coffee consumption and CHD and a 6year study of 2,975 men cited by Wilson et al. that reported, that the risk of a first cardiovascular event was decreased by $67 \%$ for those who drank more, rather than less, than 8 cups of coffee per day [15]. In addition, Ahmed et al. opposed the claim that coffee consumption increases the risk of heart failure [16].

Further studies have also indicated a beneficial effect of coffee. A recent NHANES prospective study found a strong protective association of coffee in elders without HBP. This finding was tested in the Framingham Study on 1,354 subjects aged 65-97 years. There were 210 CVD deaths and 118 CHD deaths during 10 years of surveillance. A significant $43 \%$ inverse relation between caffeinated coffee use and CHD mortality was observed for subjects with BP $<160 / 100 \mathrm{~mm} \mathrm{Hg}$. This lowered risk appears to be primarily attributable to a protective relationship between caffeinated coffee use and the onset of heart valve disease [17].

Most physicians and their patients believe that "palpitations" can result from drinking caffeinated coffee. However, after adjustment of cholesterol, BMI, BP, and other risk factors Kaiser Permanente investigators found that drinking 
more than 4 cups of coffee daily resulted in an $18 \%$ reduction in hospitalizations for cardiac rhythm disturbances in men and women, in different ethnic groups, and in smokers. An analysis of the risk reduction of decaffeinated coffee resulted in no protection, indicating that caffeine may actually be protective [18].

3.2.2. Sugar Consumption. USA sugar consumption has increased greatly in recent decades, largely due to added sugars in foods processed to increase their desirability. In an analysis of nutritional US data, Welsh et al. found a correlation between the consumption of sweeteners and blood lipid levels. This Emory University study analyzed the data of more than 6,000 men and women from 1999-2006. They found that people who add more sugar to their diet are more likely to have higher triglycerides and triglyceride/highdensity lipoprotein cholesterol ratios. Highest consumers ate 46 teaspoons of added sugars daily, the lowest 3 teaspoons daily [19]. This study, the first to examine the relation between added sugars and lipids, did not evaluate natural sugars in fruits and juices.

National surveys show that adding dietary sugar is contributing to overconsumption of calories. Johnson et al. postulate that the detrimental effect of elevated sugar consumption during a pandemic of obesity and CVD is cause for alarm. Between 1970 and 2005, dietary sugar intake increased by $19 \%$ to the current level of 22.2 teaspoons per day. The additional sugar appears to come chiefly from excessive intake of beverages. This high consumption of soft drinks replaces some essential nutrients and predisposes its association with adiposity and the "metabolic syndrome." As a consequence the American Heart Association advises limiting daily intake of sugar to less than 100 calories for women and less than 150 calories for men [20].

3.2.3. Glycemic Diet. Prospective studies of the relationship between glycemic load (GL) and glycemic index (GI) with CVD have yielded inconsistent results, especially for men. Sieri et al. investigated this issue in a 7.9 year follow-up study of 47,749 EPICOR study volunteers who had completed a nutritional survey. Over the follow-up period, 158 women and 305 men developed CHD. Women in the highest quartile of carbohydrate consumption experienced a doubling of $\mathrm{CHD}$ risk compared to those in the lowest quartile. However, the same analysis in men yielded no association. Excess intake of carbohydrate foods with a high-glycemic index raises the blood sugar level and increases insulin resistance. This imposes a significant 1.7-fold excess of CHD for women only. For those in the highest quartile as opposed to those in the lowest quartile CHD risk was increased more than 2 -fold. It is postulated that the adverse effects of a high glycemic diet in women may be attributable to sex differences in lipoprotein and glucose metabolism [21].

Switching to a low glycemic diet can be relatively easy. As recommended by the Glycemic Index Foundation Sydney Australia, the basic premise is to substitute high glycemic index carbohydrates for low glycemic carbohydrates. This entails use of oats, barley and bran in breakfast cereals; eating wholegrain breads and cutting down on the amounts of potatoes consumed. All other types of fruits and vegetables can be savored along with basmati rice, pasta, noodles, quinoa and salad vegetables with a vinaigrette dressing [22].

3.2.4. Chocolate. Recent data suggest that eating chocolate may actually lower blood pressure and risk of CVD. In a large European study of more than 19,000 volunteers, Buijsse et al. found that participants eating 7.5 grams of chocolate daily had a lower risk of myocardial infarction and stroke than those who consumed only $1.7 \mathrm{~g}$ of chocolate per day. During the 8 years of the study, there were 166 myocardial infarctions and 136 strokes. The top quartile of chocolate consumers had a $27 \%$ reduced risk of myocardial infarction and $48 \%$ lower risk of stroke than the lowest quartile chocolate consumers. The combined outcome of myocardial infarction and stroke was reduced 39\% $(P=.01)$. Chocolate and cocoa have a marked effect on blood pressure and in this study the lower blood pressure induced accounted for $12 \%$ of the reduction in CVD risk. However, even after adjusting for blood pressure, those in the top chocolate consumption quartile still had a $32 \%$ decreased risk of CVD compared to those in the bottom quartile [23].

To date, there is no established recommendation for healthful chocolate consumption for the purpose of preserving cardiovascular health. Flavonoids in cocoa oppose free radical injury because of their antioxidant effect. They also decrease cholesterol, lower blood pressure, inhibit sticky platelets, and improve blood flow to vital organs. During the processing of cocoa into chocolate some of its beneficial flavonoids are lost. Adding dark chocolate to the diet appears preferential to eating milk chocolate. However, despite the potential health benefits, one must recognize that $100 \mathrm{~g}$ of dark chocolate has 500 calories, which must be taken into account if weight gain is to be avoided. Presently, it appears prudent to enjoy moderate one ounce portions of chocolate several times a week. Other options for supplementing the diet with flavinoid-rich foods are apples, cranberries, grapes, onions, peanuts, orange juice, red wine, and tea [24].

3.2.5. Nut Consumption. It appears that eating nuts as part of a balanced diet may favorably affect blood lipids and potentially lower the risk of CHD. Epidemiological studies indicate that the risk of $\mathrm{CHD}$ is $40 \%$ lower in persons who eat more than 4 servings of nuts per week as opposed to those who do not eat any. A meta-analysis of 25 studies undertaken by Sabaté et al. examined whether the effects of eating nuts on blood lipids vary by population, type of nuts consumed, diet, or BMI in untreated subjects with normal lipids and blood pressure. The results of this analysis indicate that consuming $67 \mathrm{~g}$ of nuts daily reduced total cholesterol by $10.9 \mathrm{mg} / \mathrm{dL}$ and LDL-C $10.2 \mathrm{mg} / \mathrm{dL}$. It also significantly improved the ratios of LDL/ HDL cholesterol and total/HDL cholesterol. However, there was no significant effect on HDL$\mathrm{C}$ or triglycerides per se. The risk effect was similar in both sexes and all age groups, and it was observed regardless of the specific nut consumed. Lipid lowering was greater for persons with higher LDL-C $(18.4 \mathrm{mg} / \mathrm{dL})$ than lower LDL-C $(3.5 \mathrm{mg} / \mathrm{dL})$ and $\mathrm{BMI}<25[25]$. 
3.2.6. Orange Juice. A new study claims that drinking orange juice with meals can counter the proinflammatory effects of high-carbohydrate, high-fat meals. High carbohydrate, high fat meals induce a protein, suppressor of cytokine signaling 3 (SOCS-3), that interferes with the action of insulin promoting insulin resistance. These adverse changes can be avoided when orange juice is consumed with a meal. This implies that drinking orange juice could potentially help prevent insulin resistance, diabetes, CHD and strokes. This benefit may be attributable to the flavinoids in orange juice, which suppress reactive oxygen metabolites [26].

3.2.7. Vitamin $D$. New studies support the link between vitamin D deficiency and CVD [27-35]. Bair et al. observed 9.491 persons with low-level vitamin $\mathrm{D}$ and those who normalized D levels after one year comparing outcomes in CHD, MI and heart failure, stroke, renal failure, and death. They found that those who normalized vitamin D levels were less likely to develop heart failure or CVD, and they were less likely to die [27]. The effect of vitamin $\mathrm{D}$ was quantified in a study by May et al. who divided 31289 patients into 3 levels and linked them with several adverse outcomes, mostly CVD. An inverse relationship was found between increasing vitamin D levels and adverse events. Optimal vitamin D was defined as greater than 43 nanograms per milliliter $(\mathrm{ng} / \mathrm{mL})$ [28]. Penckofer et al. indicate a further benefit showing that adequate intake of vitamin $\mathrm{D}$ may impede diabetes. They postulate that vitamin D may aid in the regulation of metabolic factors via beta-cell operation [29].

Vitamin D deficiency also appears to lead to high blood pressure. In a study of premenopausal women with deficient vitamin D levels, Griffin et al. found that after 15 years, women whose vitamin $\mathrm{D}$ levels remained deficient, (less than $80 \mathrm{nmol} / \mathrm{L}$ ), had a three times greater risk of developing hypertension than those women whose vitamin D levels were normal [30]. In the Framingham Study, Wang et al. found that persons with $>15 \mathrm{ng} / \mathrm{mL}$ versus $<15 \mathrm{ng} / \mathrm{mL}$ vitamin $\mathrm{D}$ had a multivariable adjusted hazard ratio of $1.62(P<.01)$ for new CVD, but only in hypertensive subjects (HR 2.13) [31]. Evidently the health benefits of vitamin $\mathrm{D}$ go beyond strong bones, protecting against CVD and even boosting the immune system [32].

However data reported on utility of vitamin D supplements for the treatment, prevention, and reversal of many health conditions such as hypertension, diabetes, obesity, and CVD are inconsistent. In a systematic review of 5 prospective studies of dialysis patients and one general population study of subjects receiving vitamin D supplements, Wang et al. found a decrease in CVD deaths [33]. However, Pittas et al. found the association between vitamin D status and cardiometabolic outcomes to be uncertain. Trials they reviewed showed no clinically significant effect of vitamin D supplementation [34]. Further studies may be necessary to determine an optimum dosage of vitamin $\mathrm{D}$.

Meanwhile, increasing vitamin $\mathrm{D}$ levels appears to be necessary. Small amounts of vitamin D can be obtained from foods such as fish and mushrooms as well as from vitamin supplements. However, obtaining vitamin D from sun exposure has a greater duration of effect. Although, physicians usually advise patients to avoid unprotected sun exposure, its beneficial effects are essential, albeit in moderation. On exposure to the sun, the skin produces vitamin $\mathrm{D}$ and preserves it in adipose tissue. Vitamin D is then released when sunlight is limited such as in winter months in the Northern Hemisphere. Ten minutes of sun exposure daily is not only safe, but necessary for optimal health. In person over 65 years of age and for residents living in areas where sun exposure is limited, a dietary supplement is recommended $[29,35]$.

3.3. Portion Control for Weight Management. Portion control is essential for weight loss and for maintaining a healthy weight. In 2007, Pederson et al. completed a randomized trial of weight control in 130 participants with type 2 diabetes. The group using a portion-control plate decreased their body weight by $1.75 \%$ versus a $0.05 \%$ weight loss in the group who did not. The group using the portion-control plate also decreased use of medication for the management of diabetes, suggesting that portion-control plates are as effective as costly diet medication [36]. The diet plate is a commercially available plate with recommended foods and serving sizes for a healthful diet depicted on it.

When presented with greater portion sizes, people tend to eat more but report the same level of satiation. In a study by Rolls et al. participants were given four different portion sizes of macaroni and cheese on different days. Although, consumption increased with the portion size served, participants reported similar levels of satiation and less than half noticed that portion sizes had changed. Consumption of the largest portion as opposed to the smallest portion of macaroni amounted to $30 \%$ more energy (162 cal) [37]. In a similar study, the same participants were given different sizes of subsandwiches on 4 different days. The participants ate significantly more as the sandwich size increased [38] Rolls et al. also examined how energy intake increases in both men and women as the portion size of a snack increases. On 5 different occasions, they gave men and women bags of potato crisps that appeared to be similar in size but the quantity varied from $28 \mathrm{~g}$ to $170 \mathrm{~g}$. As the package size increased the amount of consumption also increased [39]. A study by Diliberti et al. showed similar effect in that participants who were served different quantities on different days in a restaurant, increased consumption as portion sizes increased [40]. Increased portion size appears to make it difficult for people to determine how much is being consumed and leads to excess calorie consumption. Therefore, public awareness of the effect portion size can have on weight control efforts is essential [41].

3.4. Alcohol and Cardiovascular Disease. Meta-analyses of experimental and observational studies indicate that moderate consumption of alcohol is beneficial for decreasing the risk of CHD. Published research has for many years suggested a link between alcohol consumption and reduced CVD mortality. Alcohol is associated with a modest increase in HDL cholesterol and may play a role in reduction of thrombus formation which reduces risk of stroke and CHD. A study examining the effects of alcohol on cholesterol and 
haemostatic factors by Rimm et al. showed that consumption of $30 \mathrm{~g}$ of alcohol per day can reduce risk of CHD by $24.7 \%$ [42]. Some speculate that the benefits may derive from the beneficial antioxidants and flavonoids contained in red wine. However, it is unclear if the type of alcohol consumed, or other healthy behaviors of those who consume alcohol in moderation, is responsible for the reduced risk [43]. Nonetheless, Mukamal et al. found that moderate alcohol consumption appears to confer a lower risk of myocardial infarction even in men who were already determined to be at low risk of MI based on their healthy lifestyle choices and BMI [44]. Likewise, Sesso et al. found a significant reduction in risk of $\mathrm{CHD}$ in men who increased consumption of alcohol from very low to moderate levels. The study included 18,455 men who were tracked over a period of 7 years [45].

The AHA reluctantly recommends moderate consumption of no more than two drinks per day for men and one drink per day for women. A drink is one $12 \mathrm{oz}$. beer, $4 \mathrm{oz}$. of wine, $1.5 \mathrm{oz}$. of 80 -proof spirits, or $1 \mathrm{oz}$. of 100 -proof spirits. It reminds us that drinking more alcohol than recommended is associated with alcoholism, accidents, suicide, cirrhosis of the liver, and breast cancer. With consideration of the detrimental effects, the AHA does not recommend that abstainers start drinking alcohol [46].

\section{Discussion}

4.1. Magnitude of the Cardiovascular Threat. The Framingham study has determined the actual lifetime risk of developing atherosclerotic cardiovascular disease from a general population sample examined biennially since 1950 . In the 60 years of surveillance by routine examination of the population, the lifetime risk of developing a cardiovascular event for a 50 -year-old was $52 \%$ for men and more than $39 \%$ for women with little or suboptimal treatment [4750]. Ford et al. estimate that half the reduction in USA CHD mortality since 1980 is attributable to cardiovascular risk factor reduction in the population [51] Thus, correction of risk factors discovered by the Framingham study has resulted in a major reduction in CHD deaths in the US population; $64 \%$ for non-CHD deaths and 49\% for sudden deaths [52]. A faulty diet is in part responsible for many of the major CVD risk factors including dyslipidemia, hypertension, obesity, impaired glucose tolerance, and diabetes.

4.2. Lifestyle and Behavior. The USA allocates more funds for its medical system than any other country but performs poorly on all measures for the quality of health care. In comparison with 30 developed nations of the world, the US trails behind on most standard measures of quality health care. Reports based on 2004 data from 192 nations, placed the USA at 46th in average life expectancy and $42^{\text {nd }}$ in infant mortality. Whereas a mere $10 \%$ of the population's mortality is attributable to the quality of health care and $40 \%$ is attributable to detrimental behaviors, the best means for improving health and reducing premature death may lie in the modification of personal behavior $[53,54]$. Physicians need to place greater emphasis on modifying patient's CVDpromoting behavior and among these, a more healthy diet warrants a high priority.

\section{Conclusion}

A healthy lifestyle for avoiding CVD and its predisposing risk factors and thus prolonging lifespan must include a healthy diet that has many features of a Mediterranean or Oriental cuisine. Recent trials and data suggest that certain nutrients formerly indicted as harmful need not be avoided including moderate consumption of eggs, chocolate, nuts, and caffeinated coffee, among others. Recommendations for prevention of CVD have much in common regarding CVD risk factors. Nutritional risk factors appear to apply for more than dyslipidemia. For hypertension recommendations include a lean body mass, restricted sodium intake, and a diet that emphasizes fruits and vegetables, and low-fat dairy products. For Diabetes recommendations include curbing weight gain, control of blood pressure and lipids by eating a balanced diet. For a reduced HDL-C one should also control weight and eat fatty fish (e.g., salmon, sardines, and tuna). For Obesity requirements include control of food portion size. For a healthy $L D L-C$ one must eat less saturated fat, trans fatty acids, fewer calories and cram up on whole grains (at least 3 servings a day) eat 6 ounces of fattier fish and gorge on fruits, vegetables, and legumes (6 servings a day). Dietary lifestyle and behavior modification can play a major role in avoiding and correcting risk factors for CVD a major determinant of longevity.

\section{References}

[1] D. E. King, A. G. Mainous III, M. Carnemolla, and C. J. Everett, "Adherence to healthy lifestyle habits in US adults, 1988-2006," American Journal of Medicine, vol. 122, no. 6, pp. 528-534, 2009.

[2] C. D. Fryar, R. Hirsch, M. S. Eberhardt, S. S. Yoon, and J. D. Wright, "Hypertension, high serum total cholesterol, and diabetes: racial and ethnic prevalence differences in U.S. adults, 1999-2006," NCHS Data Brief, no. 36, pp. 1-8, 2010.

[3] M. J. Stampfer, F. B. Hu, J. E. Manson, E. B. Rimm, and W. C. Willett, "Primary prevention of coronary heart disease in women through diet and lifestyle," New England Journal of Medicine, vol. 343, no. 1, pp. 16-22, 2000.

[4] L. Djoussé, J. A. Driver, and J. M. Gaziano, "Relation between modifiable lifestyle factors and lifetime risk of heart failure," Journal of the American Medical Association, vol. 302, no. 4, pp. 394-400, 2009.

[5] D. Mozaffarian, R. Micha, and S. Wallace, "Effects on coronary heart disease of increasing polyunsaturated fat in place of saturated fat: a systematic review and meta-analysis of randomized controlled trials," PLoS Medicine, vol. 7, no. 3, Article ID e1000252, 2010.

[6] R. Micha, S. K. Wallace, and D. Mozaffarian, "Red and processed meat consumption and risk of incident coronary heart disease, stroke, and diabetes mellitus: a systematic review and meta-analysis," Circulation, vol. 121, no. 21, pp. 22712283, 2010.

[7] R. Sinha, A. J. Cross, B. I. Graubard, M. F. Leitzmann, and A. Schatzkin, "Meat intake and mortality: a prospective study of 
over half a million people," Archives of Internal Medicine, vol. 169, no. 6, pp. 562-571, 2009.

[8] P. M. Kris-Etherton, W. S. Harris, and L. J. Appel, "Fish consumption, fish oil, omega-3 fatty acids, and cardiovascular disease," Circulation, vol. 106, no. 21, pp. 2747-2757, 2002.

[9] L. Meng, L. Wilkens, and L. Kolonel, "Fish consumption and ethnic differences in coronary heart disease mortality in a multiethnic cohort," Circulation, vol. 120, p. S498, abstract $1404,2009$.

[10] F. B. Hu, L. Bronner, W. C. Willett et al., "Fish and omega-3 fatty acid intake and risk of coronary heart disease in women," Journal of the American Medical Association, vol. 287, no. 14, pp. 1815-1821, 2002.

[11] G. M. Nair and S. J. Connolly, "Should patients with cardiovascular disease take fish oil?" Canadian Medical Association Journal, vol. 178, no. 2, pp. 181-182, 2008.

[12] T. R. Dawber, R. J. Nickerson, F. N. Brand, and J. Pool, "Eggs, serum cholesterol, and coronary heart disease," American Journal of Clinical Nutrition, vol. 36, no. 4, pp. 617-625, 1982.

[13] F. B. Hu, M. J. Stampfer, E. B. Rimm et al., "A prospective study of egg consumption and risk of cardiovascular disease in men and women," Journal of the American Medical Association, vol. 281, no. 15, pp. 1387-1394, 1999.

[14] L. Djoussé and J. M. Gaziano, "Egg consumption in relation to cardiovascular disease and mortality: the Physicians' Health Study," American Journal of Clinical Nutrition, vol. 87, no. 4, pp. 964-969, 2008.

[15] P. W. F. Wilson, R. J. Garrison, W. B. Kannel, D. L. McGee, and W. P. Castelli, "Is coffee consumption a contributor to cardiovascular disease? Insights from the Framingham study," Archives of Internal Medicine, vol. 149, no. 5, pp. 1169-1172, 1989.

[16] H. N. Ahmed, E. B. Levitan, A. Wolk, and M. A. Mittleman, "Coffee consumption and risk of heart failure in men: an analysis from the Cohort of Swedish Men," American Heart Journal, vol. 158, no. 4, pp. 667-672, 2009.

[17] J. A. Greenberg, G. Chow, and R. C. Ziegelstein, "Caffeinated coffee consumption, cardiovascular disease, and heart valve disease in the elderly (from the Framingham Study)," American Journal of Cardiology, vol. 102, no. 11, pp. 1502-1508, 2008.

[18] A. S. Hasan, C. Morton, M. A. Armstrong, N. Udaltsova, and A. L. Klatsky, "Coffee, caffeine, and risk of hospitalization for arrhythmias," American Heart Association Epidemiology and Prevention Nutrition and Physical Activity and Metabolism, vol. 461, San Francisco, Calif, USA, 2010.

[19] J. A. Welsh, A. Sharma, J. L. Abramson, V. Vaccarino, C. Gillespie, and M. B. Vos, "Caloric sweetener consumption and dyslipidemia among US adults," Journal of the American Medical Association, vol. 303, no. 15, pp. 1490-1497, 2010.

[20] R. K. Johnson, L. J. Appel, M. Brands et al., "Dietary sugars intake and cardiovascular health a scientific statement from the american heart association," Circulation, vol. 120, no. 11, pp. 1011-1020, 2009.

[21] S. Sieri, V. Krogh, F. Berrino et al., "Dietary glycemic load and index and risk of coronary heart disease in a large Italian cohort: the EPICOR study," Archives of Internal Medicine, vol. 170, no. 7, pp. 640-647, 2010.

[22] The University of Sydney, Australia, "Glycemic index and GI database," 2010, http://www.glycemicindex.com/.

[23] B. Buijsse, C. Weikert, D. Drogan, M. Bergmann, and H. Boeing, "Chocolate consumption in relation to blood pressure and risk of cardiovascular disease in German adults," European Heart Journal, vol. 31, no. 13, pp. 1616-1623, 2010.
[24] R. Corti, A. J. Flammer, N. K. Hollenberg, and T. F. Luscher, "Cocoa and cardiovascular health," Circulation, vol. 119, no. 10, pp. 1433-1441, 2009.

[25] J. Sabaté, K. Oda, and E. Ros, "Nut consumption and blood lipid levels: a pooled analysis of 25 intervention trials," Archives of Internal Medicine, vol. 170, no. 9, pp. 821-827, 2010.

[26] H. Ghanim, C. L. Sia, M. Upadhyay et al., "Orange juice neutralizes the proinflammatory effect of a high-fat, highcarbohydrate meal and prevents endotoxin increase and toll-like receptor expression," American Journal of Clinical Nutrition, vol. 91, no. 4, pp. 940-949, 2010.

[27] T. Bair, J. B. Muhlestein, H. T. May et al., "Supplementing Deficient Vitamin D levels is associated with reduced cardiovascular risk," Journal of the American College of Cardiology, vol. 55, no. 10a, p. A59.E564, 2010.

[28] H. T. May, J. L. Anderson, D. L. Lappé, B. D. Horne, T. L. Bair, and J. B. Muhlestein, "Stratifying cardiovascular risk by vitamin D levels: what are the optimal cutoffs?" Journal of the American College of Cardiology, vol. 55, no. 10a, p. A59.E563, 2010.

[29] S. Penckofer, J. Kouba, D. E. Wallis, and M. A. Emanuele, "Vitamin D and diabetes: let the sunshine in," Diabetes Educator, vol. 34, no. 6, pp. 939-954, 2008.

[30] D. Griffin, C. A. Gadebeku, and M. R. Sowers, "Vitamin D deficiency in younger women is associated with increased risk of high blood pressure in mid-life," in Proceedings of High Blood Pressure Research Conference Benefits of Vitamin D AHA, Chicago, Ill, USA, September 2009.

[31] T. J. Wang, M. J. Pencina, S. L. Booth et al., "Vitamin D deficiency and risk of cardiovascular disease," Circulation, vol. 117, no. 4, pp. 503-511, 2008.

[32] A. A. Ginde, R. Scragg, R. S. Schwartz, and C. A. Camargo Jr., "Prospective study of serum 25-hydroxyvitamin D level, cardiovascular disease mortality, and all-cause mortality in older U.S. adults," Journal of the American Geriatrics Society, vol. 57, no. 9, pp. 1595-1603, 2009.

[33] L. Wang, J. E. Manson, Y. Song, and H. D. Sesso, "Systematic review: vitamin $\mathrm{D}$ and calcium supplementation in prevention of cardiovascular events," Annals of Internal Medicine, vol. 152, no. 5, pp. 315-323, 2010.

[34] A. G. Pittas, M. Chung, T. Trikalinos et al., "Systematic review: vitamin D and cardiometabolic outcomes," Annals of Internal Medicine, vol. 152, no. 5, pp. 307-314, 2010.

[35] M. F. Holick, "Vitamin D deficiency," New England Journal of Medicine, vol. 357, no. 3, pp. 266-281, 2007.

[36] S. D. Pedersen, J. Kang, and G. A. Kline, "Portion control plate for weight loss in obese patients with type 2 diabetes mellitus: a controlled clinical trial," Archives of Internal Medicine, vol. 167, no. 12, pp. 1277-1283, 2007.

[37] B. J. Rolls, E. L. Morris, and L. S. Roe, "Portion size of food affects energy intake in normal-weight and overweight men and women," American Journal of Clinical Nutrition, vol. 76, no. 6, pp. 1207-1213, 2002.

[38] B. J. Rolls, L. S. Roe, J. S. Meengs, and D. E. Wall, "Increasing the portion size of a sandwich increases energy intake," Journal of the American Dietetic Association, vol. 104, no. 3, pp. 367372, 2004.

[39] B. J. Rolls, L. S. Roe, T. V. E. Kral, J. S. Meengs, and D. E. Wall, "Increasing the portion size of a packaged snack increases energy intake in men and women," Appetite, vol. 42, no. 1, pp. 63-69, 2004.

[40] N. Diliberti, P. L. Bordi, M. T. Conklin, L. S. Roe, and B. J. Rolls, "Increased portion size leads to increased energy intake in a restaurant meal," Obesity Research, vol. 12, no. 3, pp. 562$568,2004$. 
[41] Division of Nutrition and Physical Activity, Research to Practice Series, No. 2 "Portion Size", Centers for Disease Control and Prevention, Atlanta, Ga, USA, 2006.

[42] E. B. Rimm, P. Williams, K. Fosher, M. Criqui, and M. J. Stampfer, "Moderate alcohol intake and lower risk of coronary heart disease: meta-analysis of effects on lipids and haemostatic factors," British Medical Journal, vol. 319, no. 7224, pp. 1523-1528, 1999.

[43] T. A. Pearson, "Alcohol and heart disease," Circulation, vol. 94, no. 11, pp. 3023-3025, 1996.

[44] K. J. Mukamal, S. E. Chiuve, and E. B. Rimm, "Alcohol consumption and risk for coronary heart disease in men with healthy lifestyles," Archives of Internal Medicine, vol. 166, no. 19, pp. 2145-2150, 2006.

[45] H. D. Sesso, M. J. Stampfer, B. Rosner, C. H. Hennekens, J. E. Manson, and J. M. Gaziano, "Seven-year changes in alcohol consumption and subsequent risk of cardiovascular disease in men," Archives of Internal Medicine, vol. 160, no. 17, pp. 26052612, 2000.

[46] American Heart Association, "Alcohol, Wine and Cardiovascular Disease," 2010, http://www.heart.org/HEARTORG/ GettingHealthy/NutritionCenter/Alcohol-Wine-andCardiovascular-Disease_UCM_305864_Article.jsp.

[47] D. M. Lloyd-Jones, E. P. Leip, M. G. Larson et al., "Prediction of lifetime risk for cardiovascular disease by risk factor burden at 50 years of age," Circulation, vol. 113, no. 6, pp. 791-798, 2006.

[48] D. M. Lloyd-Jones, M. G. Larson, E. P. Leip et al., "Lifetime risk for developing congestive heart failure: the Framingham Heart Study," Circulation, vol. 106, no. 24, pp. 3068-3072, 2002.

[49] D. M. Lloyd-Jones, M. G. Larson, A. Beiser, and D. Levy, "Lifetime risk of developing coronary heart disease," Lancet, vol. 353, no. 9147, pp. 89-92, 1999.

[50] S. Seshadri, A. Beiser, M. Kelly-Hayes et al., "The lifetime risk of stroke: estimates from the framingham study," Stroke, vol. 37, no. 2, pp. 345-350, 2006.

[51] E. S. Ford, U. A. Ajani, J. B. Croft et al., "Explaining the decrease in U.S. deaths from coronary disease, 1980-2000," New England Journal of Medicine, vol. 356, no. 23, pp. 23882398, 2007.

[52] C. S. Fox, J. C. Evans, M. G. Larson, W. B. Kannel, and D. Levy, "Temporal trends in coronary heart disease mortality and sudden cardiac death from 1950 to 1999: the Framingham Heart Study," Circulation, vol. 110, no. 5, pp. 522-527, 2004.

[53] J. M. McGinnis, P. Williams-Russo, and J. R. Knickman, "The case for more active policy attention to health promotion," Health Affairs, vol. 21, no. 2, pp. 78-93, 2002.

[54] S. A. Schroeder, "We can do better-improving the health of the American people," New England Journal of Medicine, vol. 357, no. 12, pp. 1221-1228, 2007. 


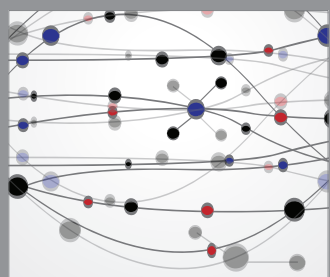

The Scientific World Journal
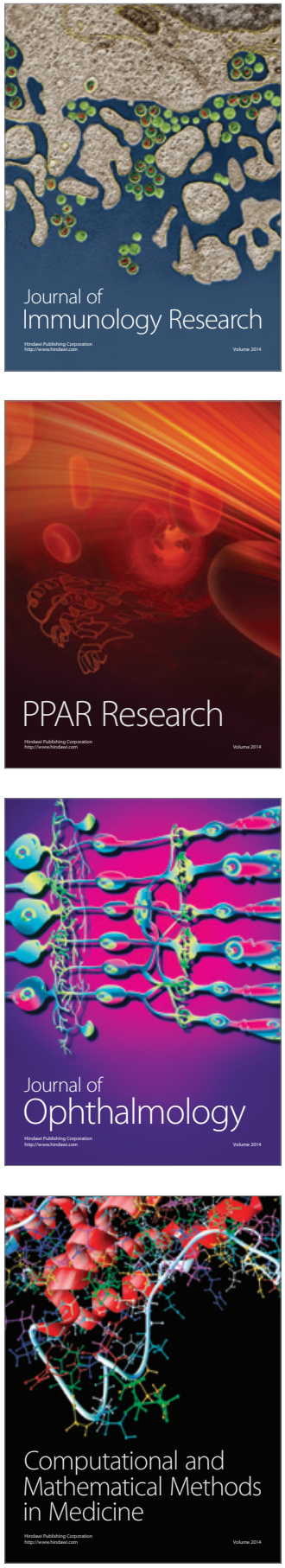

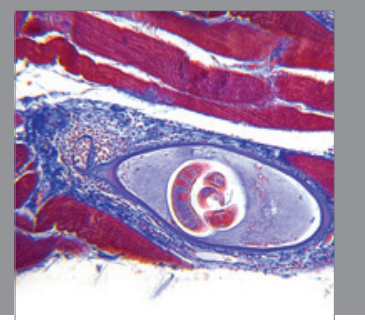

Gastroenterology

Research and Practice
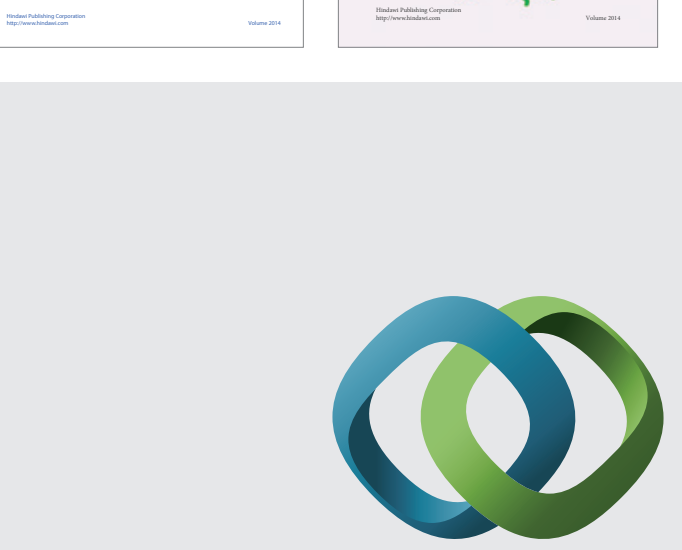

\section{Hindawi}

Submit your manuscripts at

http://www.hindawi.com
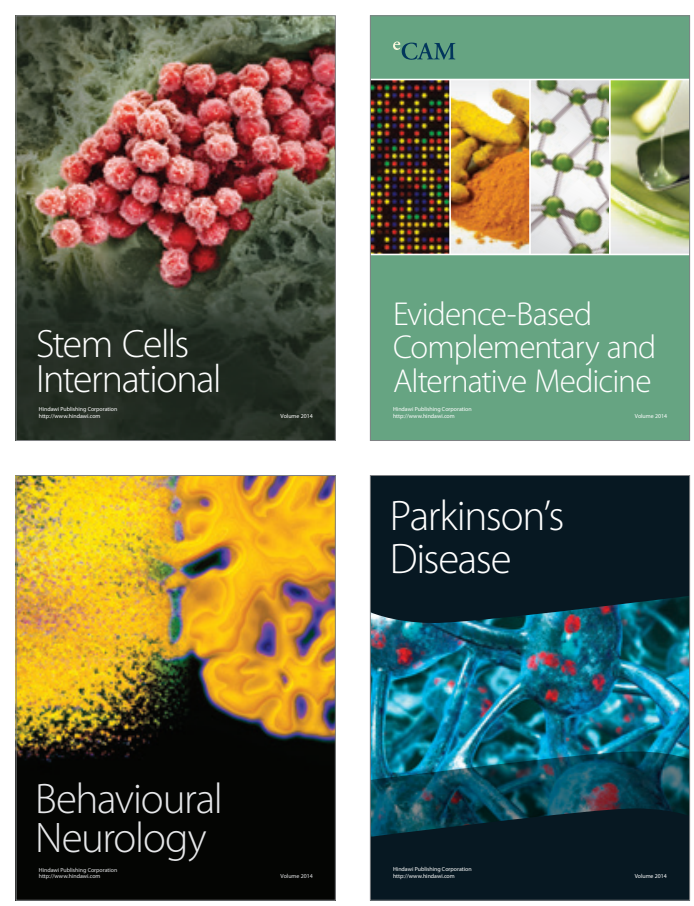

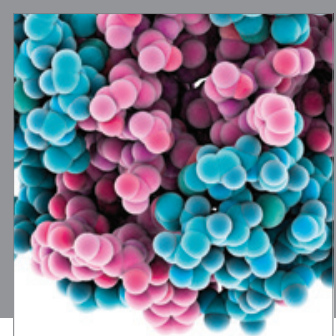

Journal of
Diabetes Research

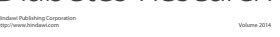

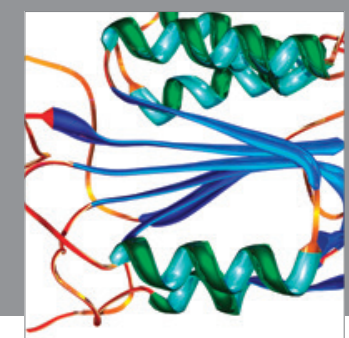

Disease Markers
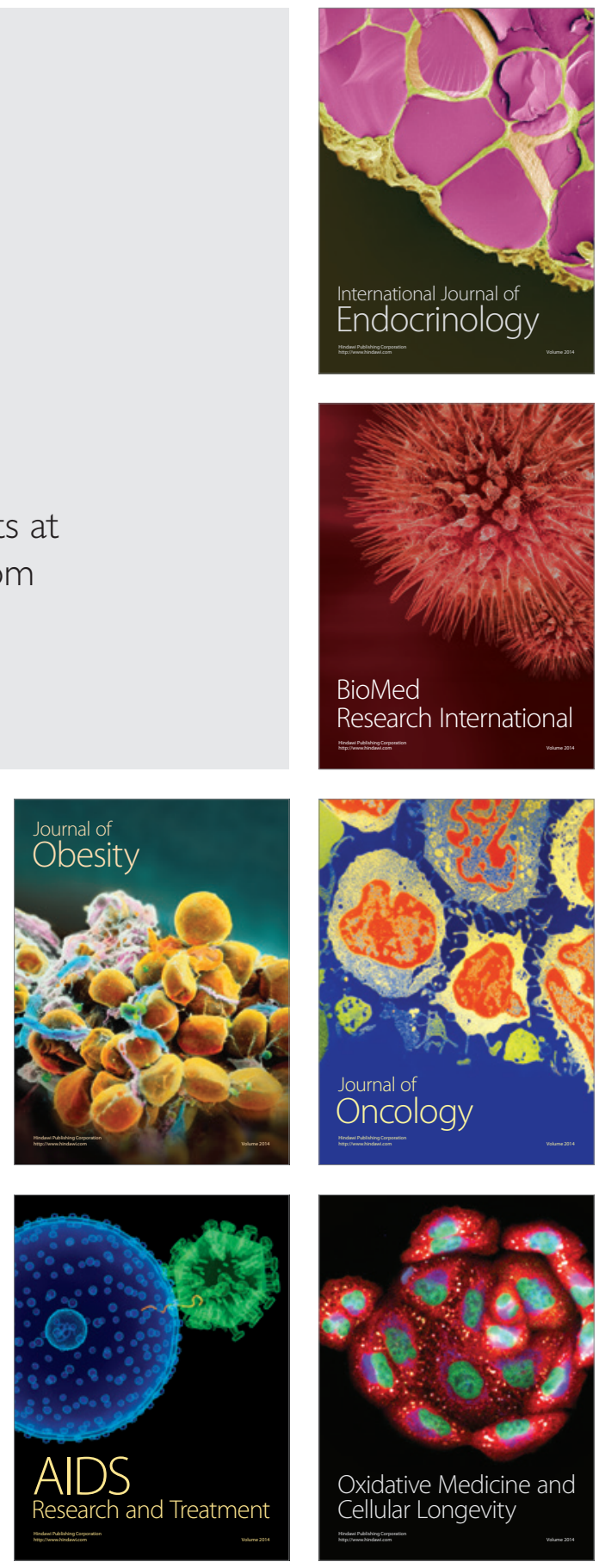